\title{
Variability in micro RNA (miRNA) abundance, speciation and complexity amongst different human populations and potential relevance to Alzheimer's disease (AD)
}

\section{Walter J. Lukiw*}

Department of Neurology, Neuroscience and Ophthalmology, LSU Neuroscience Center, Louisiana State University Health Sciences Center, New Orleans, LA, USA *Correspondence: wlukiw@lsuhsc.edu

Edited by:

Tommaso Pizzorusso, Istituto Neuroscienze CNR, Italy

Reviewed by:

Alessandro Cellerino, Scuola Normale Superiore, Italy

Ferdinando Di Cunto, University of Torino, Italy

Keywords: miRNA profiling, miRNA speciation, Alzheimer's disease, human populations, caucasian american, african american, superior temporal lobe neocortex, human biochemical individuality

Toward the end of his 1976 book entitled "Vitamin C and the Common Cold" Linus Pauling included an interesting chapter on "human biochemical individuality" that defined some important parameters on individual human genotypic versus phenotypic variation, based in part on studies from hemoglobin genetics (Pauling, 1976).That chapter provided theoretical calculations and novel insight on genomic diversity, discussing, that when any genetic characteristic is analyzed in a sampling of 100 human beings, wide ranges in values are invariably observed. The "normalcy" range was defined as that in which $95 \%$ of those "normal values" lie, while the remaining 5\% are described as "abnormal." Defining "normalcy" on a larger scale becomes a bit trickier however, and Pauling proposed that if we assume that 500 characters are independently inherited, then we can calculate that there is only a small chance, $10 \%$, that one person in the entire global population would be normal (with respect to each of these 500 characters). If we assume that there are about 26,600 human genes available to be expressed in each cell and that each gene is responsible for at least one inherited trait or genetic function (a number that is probably vastly underestimated), in a global human population exceeding 7 billion it then becomes exceedingly difficult to define "human genetic normalcy." These ideas form the basis for the evolving concept of "human genetic individuality" and our ongoing efforts to better understand the genotypic basis of human phenotypic diversity in both health and disease (Li et al., 2010; Raj et al., 2010; Lukiw, 2012a,b;
Olson, 2012; this paper). More recently, large population studies have analyzed the contribution of variability in gene expression, including the impact of genetic mutations, to "human genetic normalcy," "human genetic individuality," phenotype, susceptibility to disease and related parameters that include the general redundancy in human gene expression that may directly impact the genetic evolution of the human species (Colangelo et al., 2002; Li et al., 2010; Zheng et al., 2011; Lukiw, 2012a,b; Ginsberg et al., 2012; Raj et al., 2012; Hulse and Cai, 2013). This "Opinion paper" addresses an observed variability in micro RNA (miRNA) abundance, speciation and complexity in Alzheimer's disease $(\mathrm{AD})$, a common, progressive neurological disorder unique to the aging human brain whose incidence is approaching epidemic proportions (Alzheimer Association, 2013). Here we define miRNA abundance as how much of each individual miRNA species is present, miRNA speciation as what individual miRNA species are present, and miRNA complexity as the pattern of miRNA abundance and speciation representative of a particular physiological or pathophysiological state.

One overwhelming observation that becomes apparent in gene expression analysis is the vast variability in gene expression patterns in cells and tissues derived from different human populations - these being most noticeable from Northern-, RT-PCR- and high density array-based measurements on both messenger RNA (mRNA) and miRNA abundance, speciation and complexity in defined brain anatomical regions from different human samples. These studies have been very valuable since the profiling of mRNA and/or miRNA can provide a powerful "snapshot" into the physiological status of a human cell or tissue in health and disease, and may even be predictive for the prognosis and/or diagnosis for the future outcomes of other $\mathrm{AD}$ patients. Steady-state mRNA and miRNA levels from different individuals clearly indicates that the abundance and speciation of these RNAs within clearly defined anatomical regions can significantly differ between samples analyzed, suggesting that genetic variation and extraneous effects, including age, gender, body mass index (BMI), apolipoprotein E (ApoE), betaamyloid cleavage enzyme (BACE) and other AD-relevant allele status, life-style and intrinsic population effects can influence the profile of mRNA or miRNA abundance and complexity (Colangelo et al., 2002; Cui et al., 2005; Lukiw, 2007; Lukiw and Pogue, 2007; Williams et al., 2007; Sethi and Lukiw, 2009; Ginsberg et al., 2012). These patterns are further complicated by tissue acquisition and quality control parameters that include agonal effects, the analytical approach, and the deathto-brain freezing interval for post-mortem human tissues (McLachlan et al., 1989; Cui et al., 2005; Williams et al., 2007; Sethi and Lukiw, 2009). Agonal effects include the circumstances accompanying brain death, such as whether or not fever (i.e., heat shock) was present, whether there was interceding or accompanying illness including, commonly, pneumonia or cerebrovascular disease, and other pathophsyiological or interrelated procedural or 
clinical factors (Sethi and Lukiw, 2009; Raj et al., 2010; Hulse and Cai, 2013).

To illustrate one important example is the miRNA abundance and speciation of a small family of inducible, NF-kB-sensitive miRNAs in two different American populations-Caucasian Americans and African Americans afflicted with $\mathrm{AD}$ (Figure 1). A pathogenic quintet of upregulated miRNAs have been described and partially characterized, and these include miRNA-9, miRNA-34a, miRNA125b, miRNA-146a and miRNA-155, which have been shown to be involved in chronic inflammatory degeneration by many independent groups in multiple human diseases with a progressive inflammatory and degenerative component (Lukiw, 2007; Williams et al., 2007; Wang et al., 2009; Culpan et al., 2011; Lukiw et al., 2011; Hu et al., 2012; Iyer et al., 2012; Saba et al., 2012; Lukiw, 2013; Nussbaum, 2013; Zhao et al., 2013). In AD these five up-regulated miRNAs appear to play important roles in the down-regulation of brain gene expression normally involved in the brain's neurotrophic support, synaptogenesis, the innate-immune response, NF-kB-mediated inflammatory signaling and amyloidogenesis (Cui et al., 2005; Sethi and Lukiw, 2009; Lukiw, 2012a,b; Zhao et al., 2013). Preliminary data indicates that greater general abundance in the expression of these five miRNAs may in part explain differences in the incidence, course and/or severity of $\mathrm{AD}$ amongst elderly Caucasian American, African American, Hispanics and other minority populations. Interestingly, when comparing $\mathrm{AD}$ in human populations, African Americans and Hispanics appear to have an increased frequency and severity of $\mathrm{AD}$ when compared to Caucasians, which may be independent of their $A P O E$ genotype (Tang et al., 1998; Shadlen et al., 1999; Reitz et al., 2013). The current results further suggest that in contrast to a recent mRNA-based study of genetic homogeneity in aging humans (Colantuoni et al., 2011), increased abundance of pathological miRNAs in progressive neurodegenerative disorders may reflect gene expression patterns highly characteristic of the $\mathrm{AD}$ process in certain human populations. These results further underscore basic differences in miRNA versus mRNA function, in accordance with their differential modes of generation, processing and signaling in development, aging and disease. As both mRNA and miRNA are intrinsically unstable molecules with short half-lives, differential studies using only high quality, high RIN value, short post-mortem interval (PMI) mRNA and miRNA may be very useful in furthering our understanding of $\mathrm{AD}$ epidemiology, and ultimately also be of use diagnostically and therapeutically in the clinical management of this common neurological disorder (Espino and Lewis, 1998; Froehlich et al., 2001; Cowley et al., 2009; Sethi and Lukiw, 2009; Venketasubramanian et al., 2010; Lukiw, 2013; Reitz et al., 2013).

Variation in miRNA patterns lends further strength to the idea that $\mathrm{AD}$ is not a single, definable neurological disease entity such as sickle-cell anemia (Pauling, 1976), but rather a syndrome. Syndromes are typically a collection of biomedical symptoms known to frequently appear together, but without a known or well-defined cause. For example, the single point mutation (GAG$>$ GTG) in the beta-hemoglobin chain that changes a glutamate-to-valine, thus generating mutant hemoglobin causes sickle cell anemia virtually $100 \%$ of the time; there is no similar single nucleotide change in any gene product known that associates with, or causes AD. Rather, in AD it appears that multiple interdependent neurogenetic, neurochemical and neurobiological insults progressively accumulate and chronically drive oxidative stress, apoptosis, neuronal cell death, synaptic loss and the age-related accumulation of senile plaque and neurofibrillary tangles, the pathological hall marks of AD. Obviously neurons can die at different rates from diverse pathogenic mechanisms, and different types of neurons have varied susceptibilities and thresholds to neurotoxic insults. The recently appreciated contribution of the microbiome to human systemic physiology may very well also be involved in homeostasis, health and diseases including $\mathrm{AD}$ (Kostic et al., 2013). Further, epigenetic and environmental factors such as diet, exercise, stress and life-style, factors which are known to impact both $\mathrm{AD}$ pathology and gene expression patterns, are highly variable amongst different human populations.
Large rigorous population-based studies involving these multiple risk parameters still need to be compiled, researched and analyzed (Williams et al., 2007; Nussbaum, 2013).

Lastly, much independently derived data comparable to that shown in Figure 1 supports the idea that the genetics and epigenetics of $\mathrm{AD}$ varies widely amongst different human populations with different genetic backgrounds, and these observations are in accordance with the concept of "human genetic individuality." If molecular-genetic and epigenetic profiles of AD brain samples are any indication of $\mathrm{AD}$ phenotypic variation then there may be real and significant inter-ethnic differences in $\mathrm{AD}$ epidemiology, incidence, disease course and progression. This further suggests that an equally wide variety of diagnostic and individualistic prevention and treatment strategies will be required to more effectively address such progressive, age-related neurological disorders of the human CNS, including the implementation of novel combinatorial therapeutic strategies such as anti-NF-kB and antimiRNA approaches that have not yet been considered (Lukiw, 2013).

\section{ACKNOWLEDGMENTS}

These studies were presented in part at the 42nd Annual Society for Neuroscience Meeting, New Orleans LA, 13-17 October 2012. Sincere thanks are extended to Drs. L. Carver, E. Head, W. Poon, G. Tejada, H. LeBlanc, F. Culicchia, C. Eicken, Y. Zhao, S. Bhattacharjee and C. Hebel for short post-mortem interval (PMI) human brain tissues or extracts, miRNA array work and initial data interpretation, and to D Guillot and AI Pogue for expert technical assistance. Thanks are also extended to the many physicians and neuropathologists who have provided high quality, short PMI human brain tissues for study; additional human temporal lobe and other control and $\mathrm{AD}$ brain tissues were provided by the Memory Impairments and Neurological Disorders (MIND) Institute and the University of California, Irvine Alzheimer's Disease Research Center (UCI-ADRC; NIA P50 AG16573). The content of this manuscript is solely the responsibility of the authors and does not necessarily represent the official views of the NIH. Research on miRNA in the Lukiw 
A

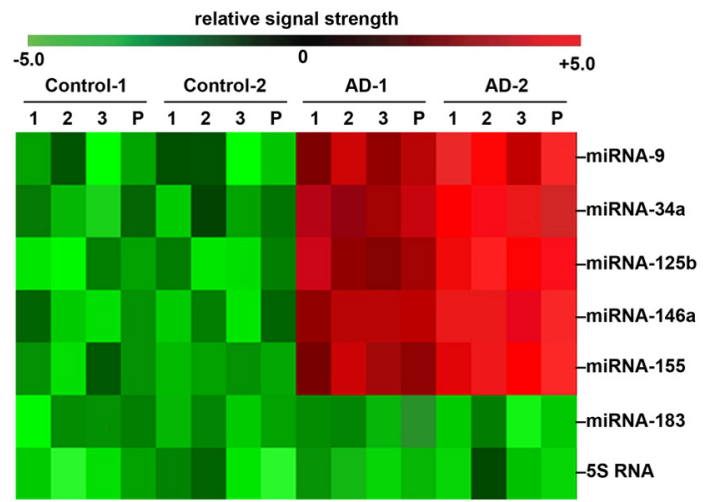

B

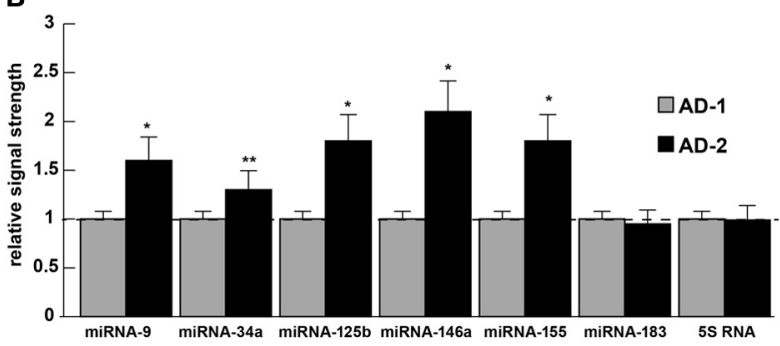

FIGURE 1 | Incidence of miRNA abundance, speciation and complexity for 5 NF-kB-sensitive pro-inflammatory miRNAs in the superior temporal lobe (Brodmann Area A22) of 2 different human populations: The current explosion in miRNA profiling of human disease, including neuro-degenerative diseases such as Alzheimer's disease (AD), underscores "human genetic individuality." Preliminary data suggests that there is considerable variation in miRNA abundance, speciation and complexity in human populations, and variation in miRNA abundance amongst individuals or populations may be a reflection of their individual genetic-based susceptibility to disease incidence or severity. (A) depicts a representative color-coded cluster diagram for 2 control and 2 selected " $A D$ " populations; control-1 miRNA signals are derived from Caucasian Americans [age mean \pm 1 standard deviation $(S D)=75.5 \pm 8.4$ year] and control-2 miRNA signals (age mean $\pm 1 S D=76.1 \pm 7.8$ year) are derived from African Americans; similarly AD-1 miRNA signals are derived from Caucasian Americans [age mean $\pm 1 S D=77.4 \pm 7.5$ year] with $A D$, and $A D-2$ miRNA signals are derived from African Americans (age mean \pm 1 $S D=76.6 \pm 8.2$ year) with $A D$; all $A D$ cases were for moderate-to-advanced stages of $A D$. Because, as single stranded ribonucleotides, miRNAs appear to have a relatively short half-life, all PMIs had a mean of $2.1 \mathrm{~h}$ or less (Sethi and Lukiw, 2009; Cui et al., 2010); there were no significant differences in age, PMI, ApoE allele status, RNA quality (all RIN values were 8.1-9.0) or yield between the control or AD groups ( $p>0.05$, ANOVA), or between the Caucasian and African American groups; note the higher general expression for miRNA-9, miRNA-34a, miRNA-125b, miRNA-146a and miRNA-155 (a) for all $A D$ cases over controls and (b) for $A D-2$ versus $A D-1$; miRNAs in all $A D$ cases were compared to 2 unchanging internal controls miRNA-183 and 5S RNA in the same brain sample; the numbers "1," "2" and " 3 " are from individual control or AD cases; the letter " $P$ " (also analyzed in B); using miRNA arrays, in Caucasian Americans miRNA-9, miRNA-34a, miRNA-125b, miRNA-146a and miRNA-155 were found to be up-regulated an average of 1.5-to-3.5 fold over age-matched controls, in African Americans this same group of miRNAs averaged an up-regulation of 3-to-5-fold over age-matched controls. (B) (bar graph) depicts quantitative results using RT-PCR, comparing AD-1 miRNA abundance $[A D,(N=8)$ relative to control miRNA $(N=8)$ signals; Caucasian Americans, set to 1.0 (for ease of comparison; dashed horizontal line)] to AD-2 miRNA abundance [AD $(N=8)$ relative to control miRNA ( $N=8$ ) signals; African Americans]; the data is suggestive of significantly higher miRNA abundance for these 5 potentially pathogenic miRNAs in the AD-2 group which may, in part, form a molecular-genetic basis for the predisposition of African Americans, and perhaps other ethnic groups, to different incidences of AD-type neuropathology, including variations in dementia development, severity, age of onset, progression, course and epidemiology (Espino and Lewis, 1998; Tang et al., 1998; Shadlen et al., 1999; Cui et al., 2010; Venketasubramanian et al., 2010; Reitz et al., 2013; this paper); ${ }^{*} p<0.01{ }^{* *} p<0.05$ (ANOVA). As further discussed in the text, selective differences in miRNA abundance may be useful in AD diagnosis and individualistic therapeutic strategies, to tailor more effective clinical treatment for $A D$ and other progressive, age-related neurological disorders of the human CNS. laboratory involving the innate-immune response in $\mathrm{AD}$, amyloidogenesis and neuroinflamamtion was supported through a COBRE III Pilot Project, a Translational Research Initiative Grant from LSUHSC, the Louisiana Biotechnology Research Network (LBRN), Alzheimer Association Investigator-Initiated Research Grant IIRG-09-131729, and NIA Grants AG18031 and AG038834.

\section{REFERENCES}

Alzheimer Association. (2013). Alzheimer's Disease Facts and Figures Statistical Resource. Available online at: http://www.alz.org/downloads/Facts Figures2013.pdf.

Colangelo, V., Schurr, J., Ball, M. J., Pelaez, R. P., and Lukiw, W. J. (2002).Gene expression profiling of 12633 genes in Alzheimer hippocampal CA1: transcription and neurotrophic factor downregulation and up-regulation of apoptotic and pro-inflammatory signaling. J. Neurosci. Res. 70, 462-473. doi: 10.1002/jnr.10351
Colantuoni, C., Lipska, B. K., Ye, T., Hyde, T. M., Tao, R., Leek, J. T., et al. (2011). Temporal dynamics and genetic control of transcription in the human prefrontal cortex. Nature 478, 19-23. doi: $10.1038 /$ nature 10524

Cowley, M. J., Cotsapas, C. J., Williams, R. B., Chan, E. K., Pulvers, J. N., Liu, M. Y., et al. (2009). Intra- and inter-individual genetic differences in gene expression. Mamm. Genome 20, 281-295. doi: 10.1007/s00335-009-9181-x

Cui, J. G., Zhao, Y., and Lukiw, W. J. (2005). Isolation of high spectral quality messenger RNA (mRNA) 
using run-on gene transcription; application to gene expression profiling of human brain. Cell. Mol. Neurobiol. 25, 789-794. doi: 10.1007/s10571005-4035-x

Cui, J. G., Li, Y. Y., Zhao, Y., Bhattacharjee, S., and Lukiw, W. J. (2010). Differential regulation of interleukin-1 receptor-associated kinase-1 (IRAK-1) and IRAK-2 by microRNA-146a and NF-kappaB in stressed human astroglial cells and in Alzheimer disease. J. Biol. Chem. 285, 38951-38960. doi: 10.1074/jbc.M110.178848

Culpan, D., Kehoe, P. G., and Love, S. (2011). Tumour necrosis factor- $\alpha$ (TNF- $\alpha$ ) and miRNA expression in frontal and temporal neocortex in Alzheimer's disease and the effect of TNF- $\alpha$ on miRNA expression in vitro. Int. J. Mol. Epidemiol. Genet. 2, 156-162.

Espino, D. V., and Lewis, R. (1998). Dementia in older minority populations; issues of prevalence, diagnosis, and treatment. Am. J. Geriatr. Psychiatry 6, S19-S25. doi: 10.1097/00019442-19982100100003

Froehlich, T. E., Bogardus, S. T. Jr., and Inouye, S. K. (2001). Dementia and race: are there differences between African Americans and Caucasians. J. Am. Geriatr. Soc. 49, 477-484. doi: 10.1046/j.15325415.2001.49096.x

Ginsberg, S. D., Alldred, M. J., and Che, S. (2012). Gene expression levels assessed by CA1 pyramidal neuron and regional hippocampal dissections in Alzheimer's disease. Neurobiol. Dis. 45, 99-107. doi: 10.1016/j.nbd.2011.07.013

Hu, K., Xie, Y. Y., Zhang, C., Ouyang, D. S., Long, H. Y., Sun, D. N., et al. (2012). MicroRNA expression profile of the hippocampus in a rat model of temporal lobe epilepsy and miR-34a-targeted neuroprotection against hippocampal neuron cell apoptosis post-status epilepticus. BMC Neurosci. 13:115. doi: 10.1186/1471-2202-13-115

Hulse, A. M., and Cai, J. J. (2013). Genetic variants contribute to gene expression variability in humans. Genetics 193, 95-108. doi: 10.1534/ genetics.112.146779

Iyer, A., Zurolo, E., Prabowo, A., Fluiter, K., Spliet, W. G., van Rijen, P. C., et al. (2012). MiRNA-146a: a key regulator of astrocyte-mediated inflammatory response. PLoS ONE 7:e44789. doi: 10.1371/ journal.pone.0044789

Kostic, A. D., Howitt, M. R., and Garrett, W. S. (2013). Exploring host-microbiome interactions in animal models and humans. Genes Dev. 27, 701-718. doi: 10.1101/gad.212522.112

Li, J., Liu, Y., Kim, T., Min, R., and Zhang, Z. (2010). Gene expression variability within and between human populations and implications toward disease susceptibility. PLoS Comput. Biol. 6:e1000910. doi:10.1371/journal.pcbi.1000910

Lukiw, W. J. (2007). Micro-RNA speciation in fetal, adult and Alzheimer's disease hippocampus. Neuroreport 18, 297-300. doi: 10.1097/WNR. $0 \mathrm{~b} 013 \mathrm{e} 3280148 \mathrm{e} 8 \mathrm{~b}$
Lukiw, W. J., and Pogue, A. I. (2007). Induction of specific micro RNA (miRNA) species by ROSgenerating metal sulfates in primary human brain cells. J. Inorg. Biochem. 101, 1265-1269. doi: 10.1016/j.jinorgbio.2007.06.004

Lukiw, W. J., Dua, P., Pogue, A. I., Eicken, C., and Hill, J. M. (2011). Up-regulation of micro RNA-146a (miRNA-146a), a marker for inflammatory neurodegeneration, in sporadic Creutzfeldt-Jakob disease (sCJD) and Gerstmann- Straussler Scheinker (GSS) syndrome. J. Toxicol. Environ. Health 74, 1460-1468. doi: 10.1080/15287394.2011. 618973

Lukiw, W. J. (2012a). NF-kB-regulated micro RNAs (miRNAs) in primary human brain cells. Exp. Neurol. 235, 484-490. doi: 10.1016/j.expneurol.2011.11.022

Lukiw, W. J. (2012b). Evolution and complexity of micro RNA in the human brain. Front. Genet. 3:166. doi: 10.3389/fgene.2012.00166

Lukiw, W. J. (2013). Antagonism of NF-кB-upregulated micro RNAs (miRNAs) in sporadic Alzheimer's disease (AD); anti-NF- $\kappa$ B versus anti-miRNA strategies. Front. Genet. 4:77. doi: 10.3389/fgene.2013.00077

McLachlan, D. R., Lukiw, W. J., and Kruck, T. P. (1989). New evidence for an active role of aluminum in Alzheimer's disease. Can. J. Neurol. Sci. 16, 490-497.

Nussbaum, R. L. (2013). Genome-wide association studies, Alzheimer disease, and understudied populations. JAMA 309, 1527-1528. doi: 10.1001/jama.2013.3507

Olson, M. V. (2012). Human genetic individuality. Annu. Rev. Genomics Hum. Genet. 13, 1-27. doi 10.1146/annurev-genom-090711-163825

Pauling, L. (1976). Vitamin C and the Common Cold WH Freeman, San Francisco CA: WH Freeman Press.

Raj, A., Kuceyeski, A., and Weiner, M. (2012). A network diffusion model of disease progression in dementia. Neuron 73, 1204-1215. doi: 10.1016/j. neuron.2011.12.040

Raj, A., Rifkin, S. A., Andersen, E., and van Oudenaarden, A. (2010). Variability in gene expression underlies incomplete penetrance. Nature 463, 913-918. doi: 10.1038/nature08781

Reitz, C., Jun, G., Naj, A., Rajbhandary, R., Vardarajan, B. N., Wang, L. S., et al. (2013). Variants in the ATP-binding cassette transporter (ABCA7), apolipoprotein $\mathrm{E} \epsilon 4$, and the risk of late-onset Alzheimer disease in African Americans. JAMA 309, 1483-1492. doi: 10.1001/jama.2013.2973

Saba, R., Gushue, S., Huzarewich, R. L., Manguiat, K., Medina, S., Robertson, C., et al. (2012). MicroRNA 146a (miR-146a) is over-expressed during prion disease and modulates the innate immune response and the microglial activation state. PLoS ONE. 7:e30832. doi:10.1371/journal.pone.0030832
Sethi, P., and Lukiw, W. J. (2009). Micro-RNA (miRNA) abundance and stability in human primary brain cells and in human brain tissues. Neurosci. Lett. 459, 100-104. doi: 10.1016/j.neulet.2009.04.052

Shadlen, M. F., Larson, E. B., Gibbons, L., McCormick, W. C., and Teri, L. (1999). Alzheimer's disease symptom severity in blacks and whites. J. Am. Geriatr. Soc. 47, 482-486.

Tang, M. X., Stern, Y., Marder, K., Bell, K., Gurland, B., Lantigua, R., et al. (1998). The APOE-epsilon4 allele and the risk of Alzheimer disease among African Americans, whites, and Hispanics. JAMA 279, 751-755. doi: 10.1001/jama.279. 10.751

Venketasubramanian, N., Sahadevan, S., Kua, E. H., Chen, C. P., and Ng, T. P. (2010). Interethnic differences in dementia epidemiology: global and Asia-Pacific perspectives. Dement. Geriatr. Cogn. Disord. 30, 492-498. doi: 10.1159/000 321675

Wang, X., Liu, P., Zhu, H., Xu, Y., Ma, C., Dai, X., et al. (2009). miR-34a, a microRNA up-regulated in a double transgenic mouse model of Alzheimer's disease, inhibits bcl2 translation. Brain Res. Bull. 80, 268-273. doi 10.1016/j.brainresbull.2009.08.006

Williams, R. B., Chan, E. K., Cowley, M. J., and Little, P. F. (2007). The influence of genetic variation on gene expression. Genome Res. 17, 1707-1716. doi: 10.1101/gr.6981507

Zhao, Y., Bhattacharjee, S., Jones, B. M., Dua, P., Alexandrov, P. N., Hill, J. M., et al. (2013). Regulation of TREM2 expression by an NF-êBsensitive miRNA-34a. Neuroreport 24, 318-323. doi: 10.1097/WNR.0b013e32835fb6b0

Zheng, W., Gianoulis, T. A., Karczewski, K. J., Zhao, H., and Snyder, M. (2011). Regulatory variation within and between species. Annu. Rev. Genomics Hum. Genet. 12, 327-346. doi: 10.1146/annurevgenom-082908-150139

Received: 13 June 2013; accepted: 06 August 2013; published online: 27 August 2013.

Citation: Lukiw WJ (2013) Variability in micro RNA (miRNA) abundance, speciation and complexity amongst different human populations and potential relevance to Alzheimer's disease (AD). Front. Cell. Neurosci. 7:133. doi: 10.3389/fncel.2013.00133

This article was submitted to the journal Frontiers in Cellular Neuroscience.

Copyright (C) 2013 Lukiw. This is an open-access article distributed under the terms of the Creative Commons Attribution License (CC BY). The use, distribution or reproduction in other forums is permitted, provided the original author(s) or licensor are credited and that the original publication in this journal is cited, in accordance with accepted academic practice. No use, distribution or reproduction is permitted which does not comply with these terms. 